hospital serving a population of 675,000 . Clinical data included demographics, laboratory parameters, aetiology and stage of liver disease. The aMAP scores were calculated using blood tests at intervals from 1-5 years prior to diagnosis of HCC to classify patients as low, medium or high risk.

Results We identified 226 patients diagnosed with HCC between 2010 and 2020. Of these, 181 (80\%) were male, median age at diagnosis was 72 years and 215 (95\%) were of white ethnicity. Aetiological factors were alcohol 78 (35\%), non-alcoholic fatty liver disease 68 (30\%), chronic viral hepatitis $54(24 \%)$ and others $20(9 \%)$. There was no prior history of liver disease in 83 (37\%). Only 56 patients (25\%) were in a formal surveillance programme and just 47 (21\%) were of Barcelona Clinic Liver Cancer (BCLC) stage A or lower at diagnosis. However, a medium or high risk aMAP risk score was identifiable prior to HCC diagnosis in $99 \%$ at 1 year, $97 \%$ at 3 years and $96 \%$ at 5 years. A high risk aMAP score alone was found in $76 \%$ at 1 year, $73 \%$ at 3 years and in $67 \%$ at 5 years prior to diagnosis with HCC.

Conclusions Our study examined a 'real world' population with HCC, representative of the broad clinical spectrum of UK liver disease. We confirmed most cases were diagnosed at a non-curative stage and were outside of a formal surveillance programme. Importantly, we have shown the vast majority of patients with HCC had demonstrably higher risk aMAP scores, which were identifiable up to 5 years before diagnosis. Use of aMAP scoring could improve targeting of candidates for HCC screening and our findings would support further, prospective evaluation in people with chronic liver disease in the UK.

\section{PWE-18 METHYLPREDNISOLONE:DIFFERENT OUTCOME IN PREDNISOLONE NON-RESPONSE SEVERE ALCOHOLIC HEPATITIS PATIENTS}

Mohamed Elnagar*, Adam Lawson, Paraskevi Mandalou, Nicholas Taylor, Andrew Austin. Royal Derby Hospital, Derby, UK

\subsection{6/gutjnl-2021-BSG.208}

Introduction Prednisolone treatment of severe alcoholic hepatitis reduces mortality from $18 \%$ to $14 \%$ at d28 (STOPAH) but not at $\mathrm{d} 90$ (30\%). Non-response may be due to steroid-resistance which might be overcome by using intravenous methylprednisolone (MePred).

Methods All patient with mDF $>32$ treated with MePred over a 4 year period were reviewed. Patients were treated with prednisolone $40 \mathrm{mg}$ daily for 7 days. Prednisolone nonresponse was defined as $\mathrm{d} 7$ Lille model $>0.45$ and severe alcoholic hepatitis confirmed by biopsy. Non-responders were given MePred $500 \mathrm{mg}$ daily for 3 days followed by prednisolone 40mg daily for 25 days and prophylactic antibiotics and fluconazole.

Results Seven non-responders were given MePred given after biopsy confirmation of alcoholic hepatitis. At d7 post-MePred, Lille score was $<0.45$ in 5 patients; one died after intracerebral haemorrhage and 4 survived beyond $\mathrm{d} 90$. Lille score remained more than 0.45 in 2 patients; one failed to respond and died at $\mathrm{d} 9$, the other died of liver failure at $\mathrm{d} 28$ despite a fall in bilirubin $\mathrm{d} 7-431$ to $\mathrm{d} 28-297$ (Figure1). No infective complications were reported. Mortality d28 29\% and d90 $42 \%$.
Bilirubin Changes During Treatment

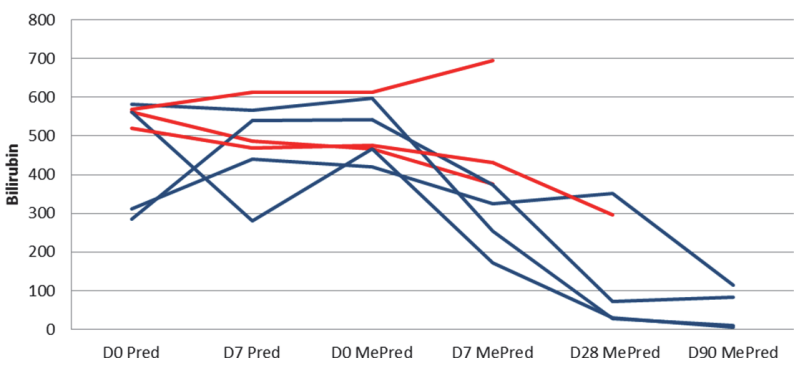

Abstract PWE-18 Figure 1 Bilirubin Changes during Treatment Key: Survivors in blue, Pred: Prednisolone

Conclusions In patients with severe alcoholic hepatitis and prednisolone non-response, methylprednisolone leads to clinical and biochemical response. Observed 6 months survival in our small cohort following MePred was $57 \%$ compared to $25 \%$ predicted by Lille model.

\section{PWE-19 THE ASSOCIATION OF EXERCISE PARTICIPATION WITH CARDIOMETABOLIC HEALTH AND QUALITY OF LIFE IN HEPATITIS C}

\begin{abstract}
1,2,3Kate Hallsworth*, 'Shion Gosrani, 'Sarah Hogg, ${ }^{1}$ Preya J Patel, 'Aaron Wetten, ${ }^{1}$ Rachael Welton, ${ }^{1,2}$ Stuart McPherson, ${ }^{4,5}$ Matthew Campbell. 'Liver Unit, The Newcastle upon Tyne Hospitals NHS Foundation Trust, Newcastle Upon Tyne, UK; ${ }^{2}$ Translational and Clinical Research Institute, Newcastle University, Newcastle upon Tyne, UK; ${ }^{3}$ Newcastle NIHR Biomedical Research Centre, Newcastle Upon Tyne, UK; ${ }^{4}$ Institute of Health Sciences and Wellbeing, University of Sunderland, Sunderland, UK; ${ }^{5}$ Leeds Institute of Cardiovascular and Metabolic Medicine, University of Leeds, Leeds, UK
\end{abstract}

\subsection{6/gutjnl-2021-BSG.209}

Introduction Hepatitis C virus infection (HCV) is associated with an increased risk of cardiovascular disease (CVD) and reduced health-related quality of life (HRQoL). Although physical activity (PA)/exercise has been shown to reduce CVD risk and improve HRQoL in patients with liver disease, there is limited data in HCV. We aimed to explore the association between PA/exercise levels, CVD risk and HRQoL in patients with $\mathrm{HCV}$ and assess individuals' attitudes to PA/exercise.

Methods Cross-sectional observational study recruiting consecutive patients with HCV from viral hepatitis clinics. Data was collected on CVD risk factors, anthropometry, HRQoL, and the exercise benefits and barriers scale (EBBS).

Results 86 patients were recruited (71\% male, 94\% white, age $52 \pm 13$ years); $49 \%$ of the cohort self-reported to be currently active. Although HRQoL was reduced across the cohort, patients that were regularly 'active' reported significantly higher HRQoL scores across SF36v2 domains compared to their inactive counterparts $(\mathrm{p}<0.05)$. Metabolic and cardiovascular characteristics were no different between groups stratified by PA/exercise status $(p>0.05)$. EBBS scores were similar in the 'active' vs 'inactive' groups, however, patients categorised as 'active' scored significantly higher on the psychological outlook and social interaction sub-scales $(p<0.05)$ than those that were 'inactive'. There were significant associations between EBBS scores and HRQoL $(p<0.05)$.

Conclusions

$\mathrm{PA} /$ exercise is associated with increased HRQoL in patients with $\mathrm{HCV}$ irrespective of clinical parameters. Addressing specific motivators/barriers to exercise for patients will be key to 
designing effective PA/exercise interventions in this patient population to ensure maximum uptake and adherence.

\section{PWE-20 PERCUTANEOUS CHOLECYSTOSTOMY RATES ARE INCREASED FOLLOWING COVID-19 INDUCED DISRUPTION TO ELECTIVE SURGICAL PATHWAYS}

Bashar Abdeen*, Paul Vulliamy, William English, K Bellam-Premnath, Ahmer Mansuri, Dipankar Mukherjee. Barking, Havering, and Redbridge NHS Trust, Romford, UK

\subsection{6/gutjpl-2021-BSG.210}

Introduction The COVID-19 pandemic has led to major service disruptions, including the cessation of elective laparoscopic cholecystectomies (LC), causing delays in managing symptomatic gallstones. We hypothesised that this would lead to an increased need for percutaneous cholecystostomy (PC) for acute cholecystitis.

Methods We performed a retrospective cohort study in a single NHS trust. We included all patients who underwent either LC or PC during the periods of March 1st - August 31st over the years 2019 and 2020. Patient data was obtained from prospectively maintained patient electronic notes. Data are presented as median and interquartile ranges for continuous data and the percentages for categorical data and compared with Mann-Whitney U-test and Fisher's exact tests respectively.

Results We observed a substantial reduction in the number of LC performed in $2020(\mathrm{n}=99)$ compared to $2019(\mathrm{n}=198)$, whilst the number of PC performed in $2020 \quad(n=35)$ was more than double that in $2019(n=17)$ (Figure.1). This increase in numbers persisted even after our LC service was restarted. Comparing the patients who underwent PC in both

\section{PERCUTANEOUS CHOLECYSTOSTOMY}

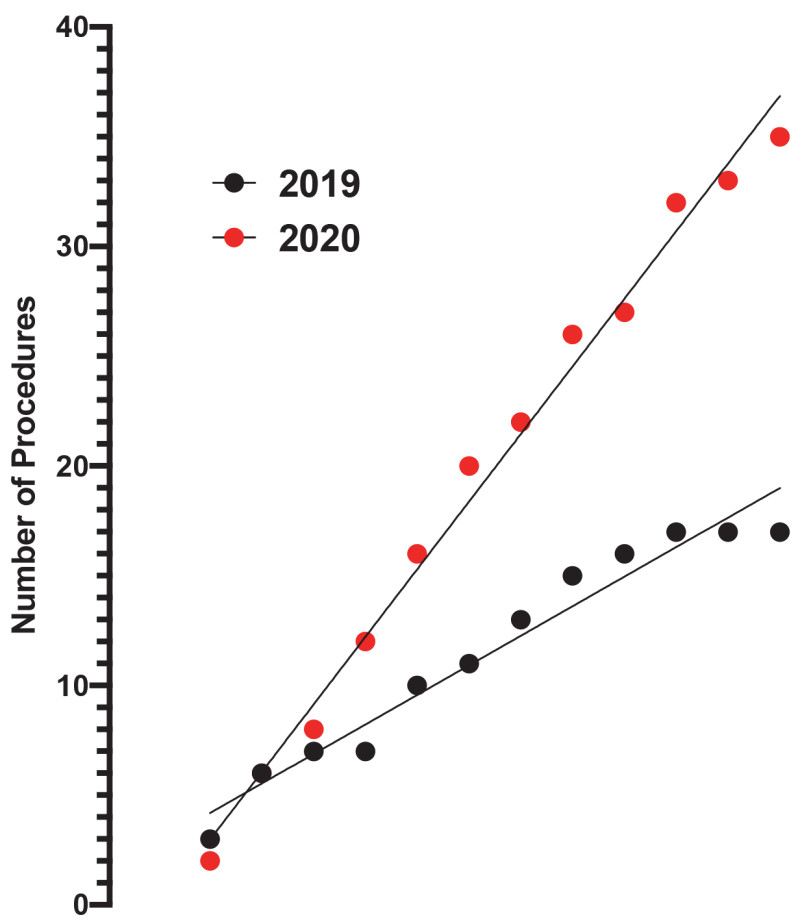

MAR APR MAY JUN JUL AUG

Abstract PWE-20 Figure 1 Percutaneous cholecystostomy years, there were no significant differences in age (2019: 68 (45-76) vs 2020: 72 (57-81), p=0.41), comorbidities (Charlson comorbidity index $\geq 4: 10 \quad(59 \%)$ vs $16(46 \%), p=0.56)$, or in-hospital mortality (2019: 2 (12\%) vs 2020: 3 (9\%), $\mathrm{p}=0.99$ ).

Conclusions These results show how the cessation of LC service was directly related to increased numbers of invasive 'damage control' procedures for acute cholecystitis, emphasising the importance of maintaining COVID-secure surgical pathways. The numbers of PC remained high even after the restart of LC service, consistent with a 'COVID shadow' resulting from interruptions to elective services that impacts patient care for a prolonged period.

\section{PWE-21 HEPATOCELLULAR CARCINOMA SURVEILLANCE: ULTRASOUND IMAGE QUALITY AND IMPLEMENTATION OF THE US LI-RADS CLASSIFICATION}

${ }^{1}$ Nicola Taylor*, ${ }^{1}$ Mandy Turner, ${ }^{1}$ Cara List, ${ }^{1}$ Hazel Allen, ${ }^{1,2}$ James Franklin, 'Earl Williams, 'Jo Tod, 'Safa Al-Shamma. 'University Hospitals Dorset NHS Foundation Trust, Bournemouth, UK; ${ }^{2}$ Bournemouth University, Bournemouth, UK

\subsection{6/gutjnl-2021-BSG.211}

Introduction 6-monthly ultrasounds (US) are offered for hepatocellular cancer (HCC) surveillance in at-risk populations. US image quality is variable, depending on patient and liver parenchymal factors, as is reporting of findings. The aim of this audit was to examine reporting practice in our institution, and the proportion of compromised surveillance ultrasounds in our large surveillance cohort.

Methods Electronic records for 50 patients with established cirrhosis undergoing HCC US surveillance were interrogated. The three most recent US were reviewed for reporting of image quality and limitations. Images from a single surveillance visit were then retrospectively reviewed and scored according to the US LI-RADS criteria by two Consultant Radiologists with more than 10 years' experience reporting abdominal US, with a third, consensus read to resolve disagreement.

Results Patients had a mean age of 66 (range 47-84; 16 females, 34 males). Mean surveillance duration was 5 years. All patients had established diagnosis of cirrhosis, most commonly due to non-alcoholic steatohepatitis (NASH) (44\%) or alcoholic liver disease (38\%). 94/149 (63\%) reports commented on quality of images or views. Terminology used for quality reporting were inconsistent amongst reporters. 25/94 (26\%) reports used terminology suggesting good or acceptable views and 16/94 (17\%) suggested significant limitation to views. The most common reasons cited for poor views were body habitus and overlying bowel gas.

Using US LI-RADS, 47 (94\%) patients were classified US-1 (negative), one (2\%) patient US-2 (subthreshold) and two (4\%) patients US-3 (positive). For image quality, 21 (42\%) patients were classified US-A (no limitations), 23 (46\%) patients US-B (moderate limitations) and 6 (12\%) patients USC (severe limitations). As expected, there were a higher proportion of severely compromised (LI-RADS US-C) examinations in patients with NASH cirrhosis vs non-NASH cirrhosis (22.7\% vs $3.6 \% ; p=0.075)$.

Discussion There was significant variability in US image quality reporting and terminology. Consistent with prior studies of HCC surveillance in Western populations, a significant proportion of patients had severely limited image quality, most 